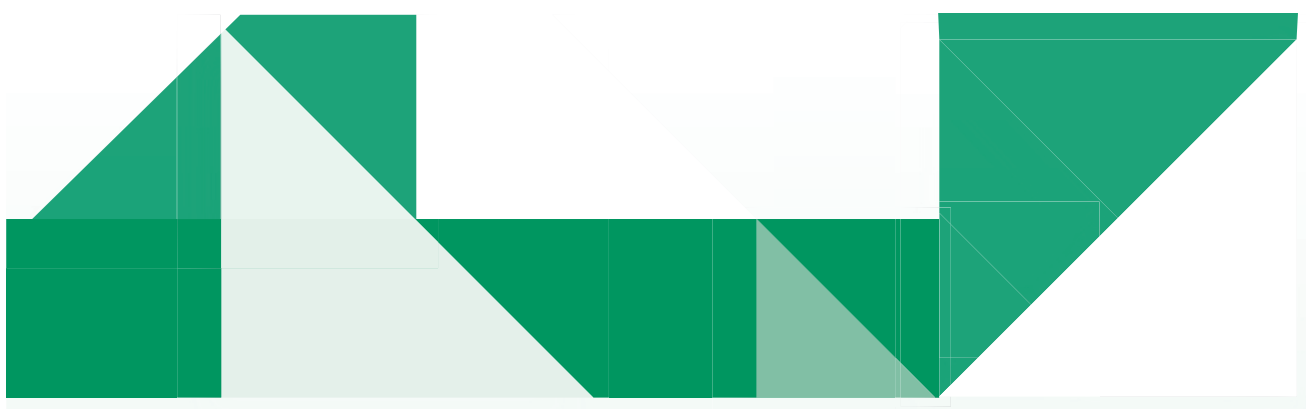

\title{
L) La reflexión y la mediación didáctica como parte fundamental en la enseñanza de las ciencias: un caso particular en los procesos de la formación docente
}

- Reflexão ensino e mediação como uma parte fundamental em Ensino de Ciências, um caso particular no Processo de Formação de Professores

- Teaching Reflection and Mediation as a Fundamental Part in Science Teaching: A Particular Case in Teacher Training Processes

\section{Resumen}

El presente artículo de investigación es el resultado de un proceso desarrollado en un curso dirigido a estudiantes de último semestre de la Licenciatura en Educación con Énfasis en Ciencias Naturales de la Universidad del Valle.

El propósito es determinar cómo la mediación didáctica y la reflexión contribuyen a los procesos de formación docente en la enseñanza de las Ciencias Naturales. Para lograrlo, se aborda la mediación didáctica, la práctica y la reflexión en la formación docente. Se destaca la importancia de las concepciones o el marco teórico de referencia del docente, así como el papel que desempeña en dicho proceso. Con ese fin, se tiene en cuenta la importancia del conocimiento disciplinar, la necesidad del conocimiento en acción o la práctica misma y la reflexión como un proceso continuo que permite fortalecer al docente en su quehacer diario.

El enfoque metodológico se basa en un estudio de caso en el cual se hace seguimiento a seis estudiantes matriculados en el curso de Mediación Didáctica, ofrecido durante un semestre, con una intensidad de 3 horas semanales, durante 16 sesiones en total. Lo anterior implica el desarrollo de cuatro momentos: (1) la caracterización académica de los estudiantes; (2) la cualificación de los docentes en formación, proceso en el cual la mediación didáctica y la reflexión desempeñan un papel fundamental; (3) el análisis de los diferentes registros

Edgar Andrés Espinosa Ríos*

Docente de la Universidad del Valle. Instituto de Educación y Pedagogía. Grupo interinstitucional Ciencia, Acciones y Creencias UPN-Uv. Correo electrónico: andres.espinosa@correounivalle. edu.co. ORCID: orcid.org/0000-0001-7637-279X 
fílmicos de los docentes en formación en su rol como docentes en el aula; y (4) el registro de la información, el análisis y las conclusiones.

Aunque el proceso de formación docente fue corto, se pueden resaltar varios aspectos. Uno de los principales factores que inciden en los procesos de enseñanza lo constituyen las concepciones pedagógicas, didácticas y disciplinares que posee el docente, pues son ellas quienes determinan su accionar en el aula. Por ello es necesario que los docentes en formación desde sus inicios como docente establezcan un equilibrio entre los contenidos curriculares y las estrategias didácticas que se plantean en el aula, para favorecer la construcción del conocimiento científico escolar.

Palabras clave

Formación docente, mediación didáctica, enseñanza de las ciencias, reflexión

\section{Resumo}

Este trabalho de pesquisa é o resultado de um processo realizado em um curso de licenciatura em educação alunos do último semestre com ênfase em Ciências Naturais da Universidad del Valle.

O objetivo é determinar como os processos de mediação do ensino e reflexão contribuem nos processos de formação de professores de ciências naturais. Para alcançar tal objetivo são aborados os processos de mediação no ensino „, prática e reflexão na formação de professores. A importância dos conceitos ou o referencial teórico do professor é realçado neste processo, tendo em conta a a importância do conhecimento disciplinar, a necessidade do conhecimento na ação ou a própria prática e reflexão como um processo contínuo que fortalece os professores no seu trabalho diário.

A abordagem metodológica é baseada em um estudo de caso no qual são estudados seis alunos matriculados no curso de mediação de ensino, que é oferecido durante um semestre, com uma intensidade de 3 horas por semana, durante 16 sessões. $\bigcirc$ desenvolvimento de foi realizado em quatro fases: a primeira,a caracterização acadêmica dos alunos, a segunda etapa envolveu a formação de professores em base da mediação do ensino e reflexão como uma parte fundamental, A terceira etapa, corresponde a análise dos diferentes registros em vídeo que são feitos na sala de aula pelos professores em formação. e o quarto e última etapa correspondeu ao registro de informações, análises e conclusões.

Embora o processo de formação de professores foi de curta duração é possível destacar vários aspectos: um dos principais fatores que influenciaram o processo de ensino esta determinado pelos conhecimentos pedagógicos, as concepções didáticas e disciplinares que possui o professor, porque elas determinam suas ações na sala de aula, segundo isto, os processos que ocorrem na sala de aula devem ser melhorados fazendo necessário que os professores em formação desde o inicio da sua formação, possam encontrar um equilíbrio entre o conteúdo curricular e as estratégias de ensino, para favorecer a construção do conhecimento científico escola.

Palavras chaves

Formação de professores, Mediação Ensino, Ensino de Ciências, Reflexão

\section{Abstract}

This research paper is the result of a process carried on a course for last semester students of the BA program in Education with Emphasis in Natural Sciences at Universidad del Valle. The goal of the study is to determine how teaching mediation and reflection contribute to teacher education processes in natural science teaching. Having this in mind, the researcher addressed the teaching mediation, practice and reflection in teacher education. It is highlighted the importance of teacher's concepts or theoretical framework, as well as their role in this process. In order to do so, I take into account the importance of subject knowledge, the need for knowledge in action or the practice itself, and reflection as a continuous process that strengthens teachers in their daily work. 
Regarding the methodology, this is a case study of six students enrolled in Teaching Mediation, a course offered 15 class hours per week, in a 16-week semester. The study took place in four stages: i) academic characterization of students; ii) qualification of pre-service teachers with didactic mediation and reflection as a fundamental component; iii) the analysis of videotaped recordings of pre-service teachers in real classroom teaching; and iv) data recording, analysis and conclusions.

Although the teacher training process was short, some aspects stand out. One of the main factors influencing teaching is composed by teacher's pedagogical, teaching and subject conceptions, since they are determining in his/her actions in the classroom. For this reason, it is necessary that since the very beginning pre-service teachers try to balance the curricular contents and teaching strategies applied in the classroom to facilitate the construction of school scientific knowledge.

Keywords

Teacher education, teaching mediation, science teaching, reflection 


\section{Introducción}

Se debe tener claro que la escuela es el espacio institucional concebido para la formación del estudiante desde dos aspectos fundamentales: la construcción de un conocimiento científico escolar a partir del desarrollo de las habilidades o competencias cognitivas y la formación del educando como un ser que es miembro de una sociedad.

El conocimiento científico escolar se construye en el aula a partir del conocimiento científico y el conocimiento cotidiano. El docente posee un conocimiento científico, que surge como resultado de su paso por las diferentes universidades, y el conocimiento cotidiano lo aporta en gran medida el estudiante fruto de su diario vivir y de su paso por diferentes instituciones educativas, sin la misma rigurosidad del docente. Esa convergencia de conocimientos que ocurre en el aula debe permitir la construcción de un conocimiento científico escolar a partir de la mediación del docente realiza, de ahí su importancia como mediador en los procesos de enseñanza y aprendizaje.

Entendiendo que el docente como mediador es el factor fundamental para que se dé la construcción de un conocimiento científico escolar, es válido preguntarse cuáles son los factores que inciden en él. Autores como Espinosa (2006) plantean que el comportamiento que el docente evidencia en el aula está dado por el marco conceptual que tiene construido, el cual determina su papel en los procesos de enseñanza y aprendizaje que ocurren en el salón de clases. Álvarez (2012) plantea que muchas de las acciones que los docentes realizan en el aula no contribuyen a los procesos de enseñanza y aprendizaje, debido a una ruptura entre la teoría y la práctica; es decir, no se vislumbra una clara relación entre el conocimiento educativo y la realidad, entre el decir y el hacer.

Dar solución a dicha ruptura no es tarea fácil. De ahí la necesidad de establecer relaciones de tipo colaborativo entre los docentes y la comunidad escolar, que permitan el trabajo a partir de contextos reales, de tal forma que el docente pueda reflexionar con mayor facilidad sobre su labor desde su práctica diaria.

Por esta razón, se debe comenzar a indagar sobre los procesos de formación docente, de tal manera que se invite a reflexionar acerca del papel que desempeñan en los procesos educativos que ocurren en el aula. A partir de lo anterior se realizó una investigación con seis estudiantes en formación de último semestre de la Licenciatura en Educación Básica con Énfasis en la Enseñanza de las Ciencias Naturales de la Universidad del Valle, en el marco del curso denominado la Mediación Didáctica en las Ciencias Naturales. Se planteó la siguiente pregunta: ¿̇Cómo la mediación didáctica y la reflexión contribuyen a los procesos de formación docente en la enseñanza de las ciencias naturales? Este interrogante orientó el trabajo realizado durante el curso y orienta el desarrollo del presente escrito, el cual refleja la experiencia y los datos recogidos. 


\section{Antecedentes}

Comúnmente se plantea la relación entre la teoría y la práctica; la existencia de una coherencia enmarcada en un contexto pedagógico; la prevalencia de una clara relación entre el pensar, el decir y el hacer; el establecer una conexión entre el conocimiento educativo y la realidad escolar en el momento de emplear los diferentes materiales didácticos en el aula. Estas conexiones y relaciones que siempre se buscan establecer en los procesos de mediación didáctica se han convertido en uno de los principales obstáculos para mejorar la enseñanza y el desarrollo profesional del docente, pues se ha observado una ruptura entre el decir (teoría) y el hacer (práctica), según lo exponen autores como Aguilar y Viniegra (2003), Montero (1997), Carr (2007), Rozada (2007), Korthagen $(2007,2010)$ y Zeichner (2010) (citados por Álvarez, 2012).

En Inglaterra, estudios realizados por Whitehead et al. (citados por Álvarez, 2012) frente a las relaciones entre la teoría y la práctica han planteado la necesidad de construir una teoría de la educación que denominan "viva" y "desde abajo", en otras palabras se desea trabajar en una realidad contextualizada y desarrollada por los propios agentes educativos. Con ello buscan satisfacer la necesidad de transformar al docente, lo cual implica que este revise su propia práctica. Se desea descubrir la teoría del docente, mediante la reflexión continua acerca de su quehacer diario.

Korthagen (2010), entre otros, ha realizado investigaciones frente a la ruptura entre la teoría y la práctica. Este autor plantea que las principales causas son la resistencia del profesorado a cambiar la forma de pensar, el poco contacto con el conocimiento pedagógico, el lenguaje empleado en el aula que en muchas ocasiones es muy formal, la poca relación entre el conocimiento disciplinar y los contextos escolares. A su vez, plantea que para mejorar dicha relación es necesario fortalecer la formación de los docentes y la capacidad de reflexión frente a sus procesos de enseñanza-aprendizaje.

La ruptura entre la teoría y la práctica se hace más visible cuando los docentes implementan los diferentes materiales didácticos en el aula. Autores como Seré (2002) muestran que muchos docentes al implementar estrategias didácticas como las prácticas de laboratorio no "invitan" a los estudiantes a reflexionar sobre la teoría aprendida, solo los llevan a comprender y aprender una teoría. Es decir, su efectividad en cuanto a la construcción del conocimiento científico escolar no radica en la herramienta misma, sino que está supeditada en gran medida a la forma y el momento en el cual el docente las emplea en los procesos de mediación.

El Ministerio de Educación Nacional (MEN) establece los estándares curriculares como orientadores para la enseñanza de las ciencias naturales. Estos son entendidos como criterios claros y públicos que permiten conocer lo que deben aprender las generaciones en formación, de manera que su aprendizaje sea pertinente para su vida y no se limiten a acumular conocimientos. Para lograr lo anterior es necesario desarrollar habilidades científicas y actitudes, con el fin de mantener la curiosidad y el beneficio de preguntar para aprender y comprender un entorno complejo y cambiante. Por eso es fundamental usar los espacios educativos para comunicar y compartir experiencias que se presentan en el entorno del estudiante. Así mismo, el MEN (2012) plantea el deseo de formar a los docentes en TIC como una necesidad de implementar estos materiales didácticos en el aula de clase. Para ello es necesario el desarrollo de competencias, teniendo bien claro que antes de esto, y de implementar cualquier 
material didáctico en los procesos de mediación en el aula, se debe promover el uso crítico desde actitudes positivas hacia la comunicación, colaboración y construcción del conocimiento.

\section{Marco teórico de referencia}

\section{Fundamentos de la mediación didáctica como parte esencial en la formación docente}

Antes de reflexionar sobre los fundamentos que orientan la mediación didáctica en el aula por parte del docente, es pertinente tener claridad en aspectos como qué se entiende por mediación y por mediador.

La mediación se debe percibir como una acción con el propósito de servir de intermediario entre las personas y su entorno, el cual se convierte en la realidad del individuo. Oliveira (1993) plantea que la mediación es uno de los conceptos fundamentales de la psicología socio-histórica, es decir de un proceso en el cual existe un agente que funciona de intermediario en una relación. Tébar (2009) plantea que el objetivo de la mediación es la construcción y el desarrollo de habilidades en el mediado (estudiante) de tal forma que lleven a su total autonomía. La mediación tiene como fundamento un principio antropológico y es la creencia de la potencialización y perfectibilidad de todo ser humano. Por tanto, se debe entender desde una posición humanizadora, constructiva y positiva enmarcada en el campo educativo, que forma parte de la capacidad de potenciar y perfeccionar al ser humano. La mediación desde un contexto educativo involucra al educador o toda persona que facilita el desarrollo, quien se convierte en un intermediario entre el estudiante y el saber, el estudiante y su entorno, y con otros pares (estudiantes).

El mediador será aquella persona que se convierte en el amplificador, intermediario, adaptador, organizador y diseñador de los procesos formativos del estudiante. El mediador deberá regular y favorecer los procesos de aprendizaje, a partir del acompañamiento y la evaluación constantes, concebida esta última como un seguimiento formativo que brinde herramientas que permitan favorecer dicho proceso (Hadii, 1992, citado por Tébar, 2009). Lo anterior implica que el mediador se interponga entre los estímulos externos, los interprete, los valore $y$, de ser necesario, los modifique antes de que sean percibidos por los estudiantes. De tal forma tendrá garantía de que el estímulo contribuirá en gran medida al aprendizaje en los estudiantes, pues es él (mediador) la persona que cuenta con los conocimientos disciplinares y pedagógicos que le permitirán orientar dicho proceso. Lograr lo anterior implica que el mediador sea el primer modificado, y se convierta en la persona que más necesita de "automodificación"; así podrá llegar al estudiante, pues una ausencia de mediación genera una privación cultural y un posible subdesarrollo 
de las capacidades del individuo (Avanzini, 1992, citado por Tébar, 2009).

Fuerstein (1996) retoma el planteamiento de Piaget en cuanto al desarrollo cognitivo en función de la interacción entre el estímulo, el organismo y la respuesta e introduce la acción mediadora, la cual se interpone entre los estímulos y el organismo, entre el organismo y la respuesta (véase la figura 1).

\section{$S \equiv H \cong O \leftleftarrows H$}

Figura 1. La experiencia de aprendizaje mediado (EAM) (Tébar, 2009).

$\mathrm{S}=$ estímulo $\mathrm{O}=$ Sujeto $\mathrm{H}=$ Mediador

$R=$ respuesta

La experiencia que el estudiante inicia a partir de la mediación que el docente realiza como parte de su proceso de aprendizaje debe facilitarle la adquisición de ciertas conductas y de los aprendizajes, operaciones mentales, estrategias, significados, etc., que modificarán de una $u$ otra forma la estructura cognitiva. Esto permitirá dar respuesta adecuada a los diferentes estímulos intencionados que el mediador les proporciona, de ahí que toma gran relevancia la relación que se establece entre el estudiante y el mediador.

\section{La importancia del lenguaje y el signo en los procesos de mediación}

Vigotsky (1978, citado por Chaves 2001) plantea que el desarrollo mental es un proceso que inicia desde el exterior, el cual generalmente es una actividad que se interioriza y, por ende, se apropia. Recalca que dicha interiorización no tiene un único camino o proceso pues lo que se interioriza son las maneras históricas y culturalmente organizadas de operar a partir de las informaciones o actividades del medio. Consideraba que el lenguaje se convierte en la mayor evidencia del desarrollo cognitivo en el individuo, pues es el indicio de las funciones superiores, en la medida en que se vuelve instrumento psicológico para la regulación del comportamiento. Rego (1998) plantea que gracias a la mediación que se realiza con los instrumentos, el individuo puede establecer relaciones con el medio ambiente ya que está en contacto directo con los sistemas simbólicos que representa dicho ambiente. A partir de lo anterior se puede aseverar que el surgimiento del lenguaje es el principal mediador en cuanto a la formación y el desarrollo de funciones psicológicas superiores en la medida en que facilita la organización de los signos en estructuras complejas, lo cual trae como consecuencia el que se pueda nombrar objetos, describirlos, mencionar las cualidades y establecer vínculos entre ellos.

Rego (1998) plantea que el lenguaje adquiere gran importancia porque permite establecer algún tipo de relación con un objeto externo -el cual puede o no estar presente-, permite realizar abstracciones, analizar y generalizar características de situaciones o eventos, además de la importancia comunicativa que adquiere. Según lo anterior, el lenguaje se convierte en un sistema de mediación simbólica que permite planificar, autorregularse y comunicarse. Dicha mediación simbólica se convertirá en experiencias para el individuo 
que se interiorizarán gradualmente y en el futuro se convertirán en la conciencia que mediará entre el decir, el pensar y el actuar del individuo.

El lenguaje y los signos deberán facilitar el conocimiento de la realidad de tal forma que permitan actuar sobre ella, de ahí que se pueda mediar desde lo social, lo instrumental entre otras formas.

Tal como se ha mencionado, el lenguaje permite establecer una relación entre el mediador y el estudiante, y se convierten en indicios dotados de intencionalidad por parte del el mediador de tal forma que favorezcan el aprendizaje en el estudiante. Estos indicios se convierten en signos en el momento en que el estudiante se apropia de ellas, de tal forma que implique una reorganización en su estructura cognitiva. A partir de lo anterior el signo exigirá por parte de quien recibe la información (estudiante), una elaboración cognitiva, lo cual implica que el signo debe ser reconocido y aceptado por él para darle significado, lo que lleva a que se debe crear otro signo que supone la existencia de otro que permite entenderlo (Gutiérrez, Ball y Márquez, 2008).

Desde el pensamiento de Vigotsky el lenguaje adquiere gran importancia porque representará un signo, este se convierte en el camino para el desarrollo de procesos a nivel individual y social, por ejemplo el desarrollo de la conciencia. La conciencia se materializa en las representaciones que el sujeto hace gracias al lenguaje y a las prácticas sociales que realiza, lo que le permitirá comprender y explicar el mundo que lo rodea. Ante lo anterior, Arbeláez (2002) manifiesta "... comprender las representaciones de un sujeto implica adentrarse en su epistemología personal, en sus creencias, en sus teorías implícitas y en las representaciones sociales del contexto en el cual las ha construido y se ha construido" (p. 3).

Peirce (1987,1988; citados por Gutiérrez, et al. 2008) plantea una visión en la cual es necesaria la existencia de un intérprete, manifestando que sin significado no hay símbolo (signo), pero sin intérprete no hay símbolo (signo) (véase la figura 2). Este autor, al igual que Vigotsky, asume la existencia de una relación semiótica gracias a la existencia de un intérprete. Sisto (1998, citado por Gutiérrez et al., 2008) manifiesta que el signo no puede ser considerado como una entidad física, ni tampoco como una entidad semiótica fija, sino como el encuentro de elementos totalmente independientes.

$$
\text { Referente } \rightarrow \text { signo } \rightarrow \text { intérprete }
$$

Figura 2. Esquema planteado por Peirce $(1987,1988)$. 
La reflexión como parte fundamental en la formación docente

La actividad docente debe trascender el simple hecho de implementar ciertas estrategias en el aula. Debe visualizarse como una actividad que invite a la reflexión, de tal forma que se haga consciente el papel que desempeña el docente en el aula y no simplemente entrar en un activismo que en gran medida no fomenta el desarrollo de una autonomía por parte de los estudiantes $y$, por ende, no contribuye al desarrollo de habilidades cognitivas. De ahí la importancia de resaltar la profesionalización del maestro como una forma de mejorar las relaciones lineales que en muchas ocasiones se dan entre el estudiante y el docente, así como el activismo en el cual caen ciertos docentes, convirtiendo el ejercicio docente en una acción mecánica. Es por ello que autores como Schön (1983) se centran en el papel de la reflexión como una acción consciente que el docente debe realizar en su ejercicio, brindando así elementos que permiten comprender la actividad in situ del docente en problemas o acciones particulares.

Schön (1983) rechaza el modelo de la racionalidad técnica, que invita a asumir la práctica inteligente como una aplicación del conocimiento a las decisiones instrumentales. Recalca la existencia de un saber propio de cada individuo en la acción, un saber que surge a partir de conductas espontáneas que se hacen visibles durante la práctica, sin una operación mental previa, en algunos momentos se dice que se "actúa por instinto"; a este saber el autor lo denomina "conocer desde la acción".

Si el sentido común reconoce el saber a partir de la acción, de igual forma se reconoce que muchas veces se piensa en lo que se está realizando y en este sentido se habla de reflexionar desde la acción. La reflexión desde la acción se fundamenta en la experiencia de la sorpresa que debe dar la acción misma en el aula. Cuando la "acción por instinto" o la "acción espontánea" del docente se realiza en función de un resultado que se espera con ansiedad no lleva a pensar sobre él mismo en la medida en que ya se espera que ocurra. En cambio, si la "acción por instinto" o la "acción espontánea" del docente brinda "sorpresas agradables o prometedoras" o simplemente respuestas inesperadas, es más probable la reflexión frente a ese hecho. Por ello la reflexión se centra en gran medida y de forma interactiva en los resultados de la acción, en la acción misma y en el saber intuitivo implícito en la acción (Schön 1983).

Cuando se reflexiona desde y sobre su práctica a partir de una situación problema que no ha sido fácilmente manejable, se puede replantear y construir un nuevo marco que tratará de imponerse sobre esa situación problema. Esto permitirá que el estudiante se convierta en un investigador desde la práctica misma, y rompa aquellos esquemas, categorías y técnicas que han sido establecidas por una comunidad, de manera que la práctica y la reflexión sobre ella sean herramientas que brinden elementos para mejorar la acción del docente en el aula.

\section{Metodología}

La investigación se realiza con seis docentes en formación de la Licenciatura en Educación con Énfasis en Ciencias Naturales de la Universidad del Valle a partir de un curso ofrecido a estudiantes de último semestre, en el marco de las electivas profesionales que deben elegir los estudiantes como parte de la estructura curricular del programa. Dicho curso se ofrece durante un semestre, con una intensidad de 
tres horas semanales, por dieciséis sesiones en total. Su desarrollo implica una cualificación de los estudiantes, que incluye abordar diferentes temáticas, así como el análisis de clases de Biología y Química a cargo de docentes "expertos" ", el diseño e implementación de clases en ciencias naturales (tres clases en total a estudiantes entre los grados sexto y noveno)² y su posterior análisis.

Teniendo en cuenta lo anterior, la metodología a tener como referente será el estudio de caso. Stake (2005) plantea que: "El estudio de casos es el estudio de la particularidad y de la complejidad de un caso singular, para llegar a comprender su actividad en circunstancias importantes [...] su objetivo básico es comprender el significado de una experiencia" (p.16).

El desarrollo de la investigación implica establecer varios momentos durante la misma, tales como la caracterización académica de los estudiantes, la cualificación de los docentes en formación, el análisis de sus registros fílmicos y la anotación de la información, el análisis y las conclusiones.

El producto final será el resultado de la recolección y el análisis de la información obtenida durante el proceso, el cual se apoyará en técnicas de recolección de información tales como la filmación de clases, su observación, y la aplicación de una matriz o rejilla para su análisis, y la reflexión que los docentes en formación realizan frente a su experiencia como docentes. Lo anterior permitirá tener la información necesaria, de tal forma que su análisis permita mejorar los procesos de mediación de los futuros docentes en la enseñanza de las ciencias naturales.

\section{Diseño metodológico}

El primer momento consiste en realizar una caracterización de los estudiantes de último semestre de la Licenciatura en Educación con Énfasis en Ciencias Naturales de la Universidad del Valle. El propósito es tener la certeza de que hay homogeneidad en cuanto a su formación académica, la cual está dada a partir de la revisión del pénsum u hoja de vida académica.

El segundo momento consiste en la cualificación de los docentes en formación, el diseño, preparación e implementación de clases en ciencias naturales. Es de anotar que en este momento se analizaran diferentes clases implementadas por docentes expertos, así como las clases que implementan los docentes en formación. De esta manera el análisis y la reflexión sobre cada una de ellas permitirá construir elementos que ayudarán en la formación de los maestros estudiantes.

Schön (1983) denomina docentes expertos a aquellos docentes que tienen mucho tiempo de experiencia y reflexionan constantemente sobre su quehacer diario en busca de mejorar los procesos de enseñanza y aprendizaje.

2 La institución educativa donde realizan la práctica en el marco de los convenios con la Universidad del Valle les asigna a los estudiantes un curso de Biología o Química entre los grados sexto y noveno. Esta es la primera experiencia formal que los estudiantes tienen como futuros docentes. 
El tercer momento corresponde al análisis de las diferentes filmaciones realizadas a partir de la implementación de una matriz (véase el anexo 1). En esta se establecen tres categorías: (1) En la estructura de la clase, como su nombre lo indica, se analizan aspectos generales, tales como la estructura de la clase, la pertinencia de su secuencialidad, la coherencia entre los objetivos planteados y las actividades propuestas, etc.; (2) En los ambientes de aprendizaje se establecen los diferentes comportamientos que tiene el estudiante, los cuales han sido generados por el docente; (3) el ambiente de enseñanza hace referencia a las diferentes acciones realizadas por el docente durante el desarrollo de la clase.

El cuarto momento son las conclusiones del proceso vivido.

\section{Desarrollo del diseño metodológico}

El desarrollo del diseño metodológico se dividen en los siguientes momentos: caracterización, cualificación, planificación-ejecución y evaluación.

\section{Primer momento: caracterización en formación académica}

El programa académico de la Licenciatura en Educación Básica con Énfasis en Ciencias Naturales y Educación Ambiental de la Universidad del Valle brinda una formación básica y fundamental en las diferentes problemáticas de índole pedagógica y científica que tienen relación con la formación en las ciencias naturales y la educación ambiental en la Educación Básica. Ofrece una formación integral, la cual es una necesidad de los educadores, y profundiza sobre los contenidos, los procesos y la aplicación del saber de las ciencias naturales y la educación ambiental.
Para lograr lo anterior, el programa académico está enmarcado en los siguientes tipos de conocimientos: conocimiento socio-ambiental, conocimiento científico, conocimiento pedagógico y conocimiento didáctico. Al respecto, los estudiantes de último semestre han adquirido conocimientos básicos disciplinares en química y biología a partir de cursos como: Química I y II, Principios de Química Orgánica General y sus respectivos laboratorios; Biología Celular, Bioquímica, Fundamentos de Fisiología Humana, Zoología de Invertebrados y Vertebrados. En el conocimiento pedagógico a partir de asignaturas como Contexto Educativo y Pedagógico de las Ciencias Naturales, Contexto Curricular de las Ciencias Naturales, Modelos Pedagógicos, Líneas de Investigación, entre otros. En el conocimiento didáctico, a partir de asignaturas como: Modelos Didácticos, Historia y Enseñanza de las Ciencias Naturales, Diseño y Uso de Materiales, Diseño y Uso de Textos, Diseño y uso de Videos. Desde el conocimiento socio-ambiental a partir de asignaturas como: Ecología General, Historia de la Educación Ambiental, Cultura del Paisaje, Educación Ambiental y Desarrollo Sostenible y Problemas Ambientales. Con base en la información anterior se puede garantizar en gran medida que los estudiantes de último semestre han tenido la misma formación, lo que brinda condiciones muy similares en cuanto a la formación académica. Es de anotar que el simple hecho que los estudiantes hayan cursado y aprobado de buena forma cada uno de los cursos mencionados no significa que tengan el mismo nivel de apropiación del conocimiento dado en ellos, pero da garantía de que tienen una base conceptual similar. 


\section{Segundo momento: cualificación de los docentes en formación}

La formación docente se debe entender como un proceso de investigación que no se limita solamente a plasmarlo en un currículo; por el contrario, debe ser transformado y enriquecido a partir de la práctica misma de los docentes. Teniendo en cuenta lo anterior, se hace necesario abordar las siguientes líneas temáticas a partir del curso ofrecido a estudiantes de último semestre de la Licenciatura en Educación Básica con Énfasis en Ciencias Naturales de la Universidad del Valle:

- La Formación Docente: en ella se aborda el proceso de enseñanza-aprendizaje como construcción social; el desarrollo de competencias como una necesidad para mejorar los procesos de aprendizaje y la teoría y la práctica en la formación docente.

- La Mediación Didáctica: en este aspecto se reflexiona sobre el nuevo rol del docente (mediador y asesor); la mediación pedagógica para la autonomía en la formación docente; la interacción social y la mediación como herramientas para reconceptualización de la relación desarrollo-aprendizaje; la importancia del lenguaje como parte fundamental en los procesos de enseñanza-aprendizaje; la implementación de diferentes materiales didácticos en los procesos de la mediación didáctica y la reflexión como parte fundamental en la formación docente.

- Siendo el estudiante el partícipe y ejecutor de su propio proceso educativo, la metodología que orientó el desarrollo del curso se encauzó bajo los principios de la investigación-acción pues ella permite resolver un problema real y concreto, sin ánimo de realizar ninguna generalización, con el objetivo de mejorar la práctica educativa real, en un contexto determinado.

- Se habla de un proceso planificado de acción, observación, reflexión y evaluación de carácter cíclico guiado por los agentes involucrados con el fin de mejorar la práctica educativa (véase la figura 3).

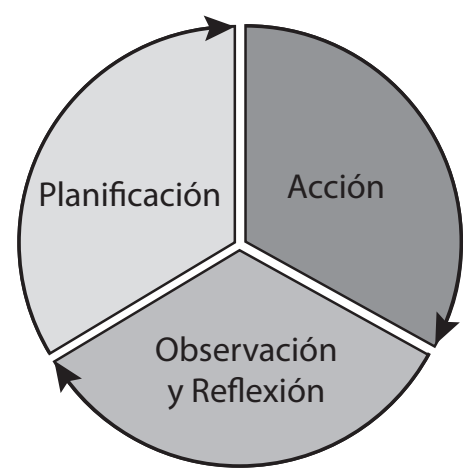

Figura 3. Caracter cíclico del proceso educativo 
En el carácter cíclico la acción se entiende como los procesos de intervención en el aula, la reflexión como los momentos en los que se analizan dichas acciones, los cuales se deben alternar de tal forma que se integren y complementen. La planificación debe ser un proceso con alto grado de flexibilidad, para poderla modificar cuando aparezcan elementos relevantes imprevistos.

Los aspectos que orientan el curso van desde lo teórico, lo práctico y la reflexión como eje transversal. Lo teórico se entiende como el momento de construcción y reconstrucción conceptual, para lo cual se realizarán seminarios y talleres de tal forma que permitan construir y reconstruir los conceptos claves en los procesos de mediación didáctica para la enseñanza de las ciencias naturales.

Este modelo de reconstrucción conceptual busca equilibrar los contenidos de las ciencias con los problemas educativos, cuando las secuencias de enseñanza y aprendizaje tienen la finalidad de mejorar su comprensión y por lo tanto favorecer el desarrollo del conocimiento científico. El modelo se puede emplear para estructurar los procesos educativos desarrollados por los docentes, puesto que ellos son parte del proceso y por lo tanto se pueden visualizar como personas integrales que aprenden, además proporciona un marco de referencia para la investigación educativa.

El segundo aspecto es el práctico, en el cual se busca que a partir del análisis de diferentes situaciones de clase, la vivencia de pensar, preparar, proponer y ejecutar una clase de ciencias naturales se afiance e interiorice. El último aspecto es la reflexión, eje transversal del desarrollo metodológico, en el cual se busca que se debatan las opiniones de los expertos acerca de la enseñanza de las ciencias a partir de las lecturas, que cada uno reflexione sobre su futuro papel como docentes y se cuestionen frente a su quehacer en ejercicio a partir de la experiencia vivida en la preparación y ejecución de una clase de ciencias naturales (véase la figura 4).

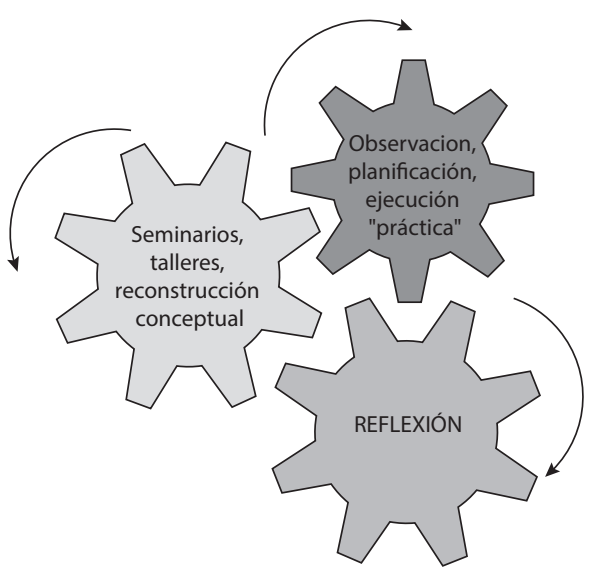

Figura 4. La reflexion como el motor del proceso educativo.

De esta forma, la reflexión genera oportunidades para que los docentes en formación y en ejercicio expliciten sus conocimientos, creencias y valores acerca de la enseñanza y aprendizaje de las ciencias. Además, les brinda experiencias que les facilitan el comenzar a clarificar, enfrentar y posibilitar la evolución progresiva de su sistema de conocimientos, creencias y valores (Abell y Bryan, 1997).

Tercer momento: planificación-ejecución y evaluación

En este momento los estudiantes en formación planifican las diferentes sesiones o clases que realizarán teniendo presente la conceptualización que van construyendo durante el desarrollo del curso, las cuales serán filmadas y analizadas como un proceso de reflexión. La importancia de este momento radica en que las estrategias metacognitivas empleadas permitan generar conciencia, de tal forma que pueda focalizar la atención en contenidos y 
acciones importantes y así se puedan alcanzar los propósitos establecidos. Al finalizar cada una de las clases realizadas por los estudiantes se implementa una matriz que permitirá realizar un análisis objetivo de ellas, el cual arrojará una valoración. De esta manera se podrá comparar el avance que obtuvieron los docentes en formación.

\section{Cuarto momento: Resultados y análisis}

A continuación se presentan los resultados obtenidos del análisis de las clases realizadas por los docentes en formación. Los valores se obtuvieron a partir de la matriz implementada y la escala utilizada para asignar la categorización (véase el anexo 1).

Tabla 1. Valoración del proceso de enseñanza de los docentes en formación

\begin{tabular}{|c|c|c|c|c|c|c|c|}
\hline \multicolumn{8}{|c|}{ Docente en formación \# 1} \\
\hline Categoría & Clase \# 1 & Valoración & \multicolumn{2}{|c|}{ Clase \# 2} & Valoración & Clase \# 3 & Valoración \\
\hline Estructura de la clase & $29 / 50$ & AS & \multicolumn{2}{|c|}{$33 / 50$} & $S$ & $32 / 50$ & $S$ \\
\hline $\begin{array}{l}\text { Ambiente de } \\
\text { aprendizaje }\end{array}$ & $15 / 35$ & AS & \multicolumn{2}{|c|}{$22 / 35$} & S & $22 / 35$ & $S$ \\
\hline $\begin{array}{l}\text { Ambiente de } \\
\text { enseñanza }\end{array}$ & $32 / 75$ & AS & \multicolumn{2}{|c|}{$44 / 75$} & AS & $43 / 75$ & AS \\
\hline \multicolumn{8}{|c|}{ Docente en formación \# 2} \\
\hline Categoría & Clase \# 1 & Valoración & $\begin{array}{l}\text { Clase } \\
\# 2\end{array}$ & Valoración & Clase \# 3 & \multicolumn{2}{|l|}{ Valoración } \\
\hline Estructura de la clase & $19 / 50$ & PS & $26 / 50$ & AS & $18 / 50$ & \multicolumn{2}{|l|}{ PS } \\
\hline $\begin{array}{l}\text { Ambiente de } \\
\text { aprendizaje }\end{array}$ & $15 / 35$ & AS & $16 / 35$ & AS & $18 / 35$ & \multicolumn{2}{|l|}{ AS } \\
\hline $\begin{array}{l}\text { Ambiente de } \\
\text { enseñanza }\end{array}$ & $26 / 75$ & PS & $32 / 75$ & AS & $20 / 75$ & \multicolumn{2}{|l|}{ PS } \\
\hline \multicolumn{8}{|c|}{ Docente en formación \# 3} \\
\hline Categoría & Clase \# 1 & Valoración & $\begin{array}{l}\text { Clase } \\
\# 2\end{array}$ & Valoración & Clase \# 3 & Valoración & \\
\hline Estructura de la clase & $35 / 50$ & $S$ & $35 / 50$ & S & $38 / 50$ & $S$ & \\
\hline $\begin{array}{l}\text { Ambiente de } \\
\text { aprendizaje }\end{array}$ & $28 / 35$ & S & $23 / 35$ & S & $24 / 35$ & $S$ & \\
\hline $\begin{array}{l}\text { Ambiente de } \\
\text { enseñanza }\end{array}$ & $50 / 75$ & S & $41 / 75$ & AS & $59 / 75$ & $S$ & \\
\hline \multicolumn{8}{|c|}{ Docente en formación \# 4} \\
\hline Categoría & Clase \# 1 & Valoración & $\begin{array}{l}\text { Clase } \\
\# 2\end{array}$ & Valoración & Clase \# 3 & Valoración & \\
\hline Estructura de la clase & $21 / 50$ & AS & $19 / 50$ & PS & $23 / 50$ & AS & \\
\hline $\begin{array}{l}\text { Ambiente de } \\
\text { aprendizaje }\end{array}$ & $11 / 35$ & PS & $13 / 35$ & PS & $18 / 35$ & AS & \\
\hline $\begin{array}{l}\text { Ambiente de } \\
\text { enseñanza }\end{array}$ & $23 / 75$ & PS & $28 / 75$ & PS & $33 / 75$ & AS & \\
\hline \multicolumn{8}{|c|}{ Docente en formación \# 5} \\
\hline Categoría & Clase \# 1 & Valoración & $\begin{array}{l}\text { Clase } \\
\# 2\end{array}$ & Valoración & Clase \# 3 & Valoración & \\
\hline Estructura de la clase & $28 / 50$ & AS & $25 / 50$ & AS & $32 / 50$ & $S$ & \\
\hline
\end{tabular}




\begin{tabular}{|l|l|l|l|l|l|l|l|l|}
\hline $\begin{array}{l}\text { Ambiente de } \\
\text { aprendizaje }\end{array}$ & $8 / 35$ & PS & $14 / 35$ & PS & $21 / 35$ & AS & \\
\hline $\begin{array}{l}\text { Ambiente de } \\
\text { enseñanza }\end{array}$ & $15 / 75$ & 1 & $25 / 75$ & PS & $42 / 75$ & AS & \\
\hline \multicolumn{7}{|c|}{ Docente en formación \# 6 } \\
\hline Categoría & Clase \# 1 & Valoración & $\begin{array}{l}\text { Clase } \\
\text { \# } 2\end{array}$ & Valoración & Clase \# 3 & Valoración & \\
\hline Estructura de la clase & $28 / 50$ & AS & $30 / 50$ & AS & $30 / 50$ & AS & \\
\hline $\begin{array}{l}\text { Ambiente de } \\
\text { aprendizaje }\end{array}$ & $16 / 35$ & AS & $15 / 35$ & AS & $20 / 35$ & AS & \\
\hline $\begin{array}{l}\text { Ambiente de } \\
\text { enseñanza }\end{array}$ & $33 / 75$ & AS & $31 / 75$ & AS & $40 / 75$ & AS & \\
\hline
\end{tabular}

Fuente: elaboración propia

El análisis de los resultados se realizará teniendo en cuenta las categorías establecidas en las rejillas, y la reflexión de los estudiantes a partir de la experiencia del curso.

A continuación se presentan los resultados obtenidos en cada una de las categorías establecidas.

\section{Categoría 1. Estructura de la clase}

Esta categoría busca analizar los aspectos generales de la clase, en ella se enmarcan subcategorías tales como los momentos que deben transcurrir durante la misma (inicio, desarrollo, cierre), la secuencialidad que se da y la pertinencia de las actividades que se plantean.

En términos generales se observa una tendencia a mejorar en dicha categoría: el docente en formación (DF) \# 2, no presentó cambio alguno; los DF \# 3, \# 4 y \# 6 presentaron una leve mejoría pero se mantuvieron en el mismo rango de valoración "satisfecho" para el DF \# 3 y "algo satisfecho" para los DF \# 4 y \# 6.

A partir de los resultados obtenidos en esta categoría se puede resaltar que las subcategorías que evidencian un mayor avance son aquellas que hacen referencia a los momentos de la clase (inicio, desarrollo y cierre), y la forma como se distribuyen los estudiantes en sus lugares de trabajo según la actividad que realizarán y la orientación del docente. No se observó un avance significativo en subcategorías como mantener un buen ambiente de trabajo, mantener un buen manejo de grupo, el manejo de un lenguaje adecuado. Es de tener presente que esta es la primera experiencia formal que los DF participantes del curso tienen, y este tipo de dificultades se comienzan a superar en gran medida a partir de las experiencias que vivan en el aula de clase.

Categoría 2. Ambientes de aprendizaje

Con esta categoría se pretende analizar los comportamientos que tiene el estudiante como respuesta a la propuesta que el docente lleva al aula. Dentro de las subcategorías se pueden mencionar: los estudiantes hacen aportes al proceso de aprendizaje, los estudiantes trabajan concentradamente y orientados hacia la resolución de las actividades, se observa una interacción entre los estudiantes con respecto al desarrollo de la clase, entre otras.

En términos generales se encuentra una mejoría; solo se observa que los DF \# 3 y \# 6 mantienen el mismo rango de valoración "satisfecho" y "algo satisfecho" respectivamente.

Se observa en gran medida avances en las subcategorías que hacen referencia a la participación y el trabajo que realizan los estudiantes en el aula de clase, lo cual permite 
inferir que los docentes en formación han apropiado y puesto en práctica lo abordado durante el curso, además de ser conscientes de su papel como docentes frente a las actividades que se realizan en el aula. Se recalca que los docentes en formación plantean como parte fundamental dentro de las actividades la participación de los estudiantes, que se convierte en el centro de aprendizaje.

Categoría 3. Ambientes de enseñanza

En ella se busca indagar sobre las acciones que el docente emplea en el aula a partir de subcategorías como la existencia de un diálogo continuo entre docente y estudiante; las actividades propuestas en el aula propician la reflexión; los métodos o estrategias planteadas permiten alcanzar los objetivos entre otras subcategorías, de tal forma que favorezcan los procesos de enseñanza y por ende la construcción del conocimiento escolar.

La tendencia en esta categoría es mantenerse en la misma valoración con la cual iniciaron el proceso; a pesar de encontrar alguna mejoría, esta no es suficiente para el cambio de indicador. Solo se observa un cambio significativo en el DF \# 5 que pasa de una valoración "insuficiente" a "algo satisfecho".

Una de las mayores dificultades que se percibe en los docentes en formación es la de no resaltar en gran medida los aportes y virtudes de los educandos al participar en la clase, y no realizar un seguimiento de las "acciones" que pueden interferir en el buen desempeño de los estudiantes en el aula.

Se resalta la mejoría de la dinámica que se desarrolla en las clases de los DF. Aspectos como el diálogo entre el docente y los estudiantes, las actividades que se proponen para propiciar su participación, la aplicación de los conocimientos pedagógicos y científicos que se vislumbran cuando se implementan en las clases, los diferentes recursos y estrategias que se usan en pro de favorecer el aprendizaje se destacan en la formación desarrollada por los DF.

Frente a las reflexiones que realizaron al finalizar el curso denominado La Mediación Didáctica en la Enseñanza de las Ciencias Naturales, los estudiantes manifestaron:

- La necesidad de tener un conocimiento disciplinar claro se convierte en fundamental en el momento de realizar la clase. El no tenerlo los obliga a no salirse de los parámetros de la clase, como consecuencia de ello el docente no podrá dar respuesta a los interrogantes planteados por los estudiantes y la suya será una clase plana.

- El ejercicio de reconocer y evaluar el desempeño docente como parte del proceso de formación genera conciencia frente al quehacer diario de la labor del docente en el aula, lo que permite construir la esencia de cada uno.

- Fortalecer la relación sujeto-signo-herramienta y objeto resulta fundamental en el proceso de la formación docente, en esa medida la corre- 
lación que se establece determinará la interpretación, el significado y la importancia de la información. De esa forma toman gran importancia los diferentes planteamientos de los pedagogos y psicólogos que fundamentan el enfoque mediador, la estimación de las ideas previas, la buena comunicación entre docente y estudiante y los espacios que se deben propiciar para la construcción del conocimiento.

\section{Conclusiones}

Teniendo en cuenta que la pregunta que orientó el trabajo de investigación fue: ¿Cómo la mediación didáctica y la reflexión contribuyen a los procesos de formación docente en la enseñanza de las ciencias naturales? se puede concluir que:

- Es importante la actitud que presenten los docentes en formación durante el proceso, pues ello determina en gran medida el avance y el alcance que se logre. Lo anterior se puede argumentar a partir del resultado obtenido por el docente \# 5, quien tuvo una actitud positiva, de compromiso y se mostró dispuesto a mejorar. Caso contrario ocurrió con el docente en formación \# 2, quien no supo sobreponerse frente a las diferentes dificultades pedagógicas encontradas; esto generó desmotivación y falta de credibilidad frente a los procesos y el papel que puede desempeñar en su rol docente.

- Los procesos de enseñanza que se llevan a cabo en el aula tienen un grado de complejidad elevado, en la medida en que es difícil tener control de todos los factores que inciden de una $u$ otra forma, pero se debe tener presente que uno de los factores que tiene mayor incidencia en dicho proceso está determinado por el docente y más aún por las concepciones pedagógicas, didácticas y disciplinares que posea, pues son ellas las que determinan su accionar en el aula. Lo anterior se vislumbra en el proceso seguido por los docentes en formación que participaron en el estudio, aunque se reconoce que tanto el tiempo de formación como la experiencia que vivieron en el aula de clase fue corto. Se observaron cambios en aspectos pedagógicos que contribuyeron a mejorar los procesos en el aula.

- En la medida en que los docentes y en especial los docentes en formación sean más conscientes de los procesos de mediación didáctica a partir de la reflexión de su quehacer diario, saliendo del mecanicismo en el cual muchos tienden a entrar, se mejorarán los procesos que ocurren en el aula. Por ello es necesario que los docentes en formación desde sus inicios establezcan un equilibrio entre los contenidos que se programan (en la estructura curricular) y las estrategias didácticas que se plantean en el aula; de tal manera se favorecerá la construcción del conocimiento escolar.

- Un aspecto importante es la actitud que tiene el docente durante los procesos de mediación; en la medida en que este incentive, oriente, reflexione y escoja experiencias en las cuales los estudiantes se vean reflejados, permitirá acceder en gran medida a la forma como el estudiante concibe y 
construye su conocimiento. Esto le brindará elementos que podrá tener en cuenta al implementar estrategias nuevas, para que se conviertan en experiencias gratas, placenteras y motivantes para los estudiantes. Este tipo de experiencias y la reflexión que el docente en formación realice sobre ellas le permitirá ser más asertivo frente a las nuevas estrategias que va a implementar, apropiándose de su rol como mediador.

\section{Referencias bibliográficas}

Abell, K. y Bryan, L. A. (1997). Reconceptualizing the Elementary Science Methods course using a reflection orientation. Journal of Science Teacher Education, 153-166.

Álvarez, A. C. (2012). La relación teoría-práctica en los procesos de enseñanza-aprendizaje. Universidad de Cantabria. Educatio Siglo xxI, 30(2), 383-402.

Álvarez, C. Á. y Maroto, J. L. S. F. (2012). La elección del estudio de caso en investigación educativa. Gazeta de Antropología, 28(1).

Arbeláez, M. (2002). Las representaciones mentales [versión electrónica]. Ciencias Humanas, 29, 1-7. Recuperado de http://www.utp.eclu.co/_chumanas/revistas/ revistas/rev29/arbelaez.htm.

Avanzini, G. (1992). Les apports du PEl dans la pensé et la practique de l'éducation. En Vv. Aa. Pédagogies de la médiation.

Espinosa, R. E. (2006). Los mediadores pedagógicos en la enseñanza de las ciencias: la implementación de un programa educativo multimedia en la enseñanza del sistema circulatorio. El Hombre y la Máquina, 32, 20-36.

Fuerstein, R. (1996). La teoría de la modificabilidad estructural cognitiva. En S. Molina, y M. Fandos. Educación cognitiva (2 vols.). Zaragoza: Mira.

Gutiérrez, M.; Ball, M. y Márquez, E. (2008). Signo, significado e intersubjetividad: una mirada cultural. Educere, 12(43), 689-695.

Hadji, Ch. (1992). Penser et agir l'éducation. Paris: ESF.

Korthagen Fred, A. J. (2010). La práctica, la teoría y la persona en la formación del profesorado. Revista Interuniversitaria de Formación del Profesorado, 68(24,2), 83-101.

Ministerio de Tecnologías de la Información y las Comunicaciones (2012). La formación de docentes en TIC, casos exitosos de computadores para educar. ISBN: 978-958-57617-0-4

Oliveira, M. K. de. (1993). Vygotsky: aprendizado e desenvolvimento um processo sócio-histórico. São Paulo: Scipione. (Série pensamento e ação no magistério).

Peirce, Ch. S. (1987). Obra lógico-semiótica. Madrid: Taurus.

Peirce, Ch. S. (1988). El hombre, un signo. Barcelona: Crítica. 
Rego, C. R. (1998). Vygotsky: uma perspectiva histórico-cultural da educação (5 ed.). Petrópolis, Rio de Janeiro: Vozes.

Schön, D. A. (1983). El profesional reflexivo. Cómo piensan los profesionales cuando actúan. Barcelona: Paidós.

Seré, M.-G. (2002). La enseñanza en el laboratorio. ¿̇Qué podemos aprender en términos de conocimiento práctico y de actitudes hacia la ciencia? Enseñanza de las Ciencias, 20(3), 357-36.

Sisto, V. (1998). Del signo al sentido. Aproximaciones para un estudio semiótico de la conciencia [versión electrónica]. Santiago: Universidad de Arte y Ciencias Sociales. Recuperado de http://168.96.200.17/ar/libros/chile/arcis/sisto.rtf.

Stake, R. E. (2005). Investigación con estudio de caso. Madrid: Morata.

Tébar Belmonte, L. (2009). El profesor mediador del aprendizaje. Bogotá: Editorial Magisterio. 


\section{Anexo 1}

Matriz para analizar las clases filmadas

El presente cuestionario es una adaptación del formato original ${ }^{3}$ de observación de clases empleado por el gobierno Alemán, el cual tiene como base el marco de calidad de los colegios alemanes en el extranjero. La presente adaptación busca realizar un análisis del papel del docente como mediador didáctico en el aula de clase, de tal forma que brinde elementos dentro del proceso de formación docente en Ciencias Naturales y Educación ambiental.

Fecha: Asignatura: Grado: Institución educativa: Privado...... Oficial....... Institución educativa: Docente(s):

\begin{tabular}{|c|c|c|c|c|c|}
\hline $\begin{array}{l}\text { Aspecto a tener en cuenta } \\
\text { Valoración }\end{array}$ & 1 & 2 & 3 & 4 & 5 \\
\hline Estructura de la clase & & & & & \\
\hline Inicio: se establecen los propósitos que se desean alcanzar durante la clase. & & & & & \\
\hline $\begin{array}{l}\text { Desarrollo: Se realizan actividades que permitan alcanzar los propósitos } \\
\text { establecidos. }\end{array}$ & & & & & \\
\hline $\begin{array}{l}\text { Cierre: el docente hace un cierre de forma adecuada estableciendo las } \\
\text { conclusiones o ideas finales de la actividad. }\end{array}$ & & & & & \\
\hline $\begin{array}{l}\text { Los objetivos de la clase se mencionan al inicio de la misma y estos son aclarados } \\
\text { o explicados. }\end{array}$ & & & & & \\
\hline Se observa una secuencia clara y pertinente de las fases de la clase. & & & & & \\
\hline Se identifican claramente el inicio y el final de la clase. & & & & & \\
\hline $\begin{array}{l}\text { El profesor vela por un buen ambiente de trabajo, es decir, por mantener el ruido en } \\
\text { un nivel adecuado y por qué los estudiantes puedan participar y ser escuchados. }\end{array}$ & & & & & \\
\hline $\begin{array}{l}\text { Si se interrumpe la clase (por tardanzas, conflictos entre alumnos, etc.), el profesor } \\
\text { interviene con rapidez y determinación. }\end{array}$ & & & & & \\
\hline Se observa un manejo de grupo que permite el buen desarrollo de la clase. & & & & & \\
\hline $\begin{array}{l}\text { El lenguaje del profesor durante la clase es adecuado para la clase y facilita la } \\
\text { comprensión del tema a desarrollar. }\end{array}$ & & & & & \\
\hline $\begin{array}{l}\text { Los estudiantes demuestran el manejo de las rutinas durante el trabajo en equipo y } \\
\text { en parejas. }\end{array}$ & & & & & \\
\hline $\begin{array}{l}\text { La distribución de los puestos de trabajo es acorde al tipo de actividades que se } \\
\text { realizan en el aula. }\end{array}$ & & & & & \\
\hline Ambientes de Aprendizaje & & & & & \\
\hline $\begin{array}{l}\text { El proceso de aprendizaje se construye con base a los conocimientos previos, } \\
\text { habilidades y la orientación dada por el docente. }\end{array}$ & & & & & \\
\hline $\begin{array}{l}\text { Los estudiantes establecen relaciones con lo abordado en clases anteriores u otras } \\
\text { disciplinas. }\end{array}$ & & & & & \\
\hline $\begin{array}{l}\text { La situación de aprendizaje aporta al ámbito de realidad de los alumnos } \\
\text { (Aprendizaje significativo/Aprendizaje cercano a la realidad). }\end{array}$ & & & & & \\
\hline Los estudiantes hacen aportes al proceso de aprendizaje. & & & & & \\
\hline $\begin{array}{l}\text { Los estudiantes trabajan concentradamente y orientados hacia la resolución de las } \\
\text { actividades. }\end{array}$ & & & & & \\
\hline
\end{tabular}


Se observa una interacción entre los estudiantes con respecto al desarrollo de la clase.

Los estudiantes utilizan los medios de manera adecuada: como fuente de información (p. ej. libro de texto, utensilios experimentales, computador, calculadora de bolsillo, diccionarios).

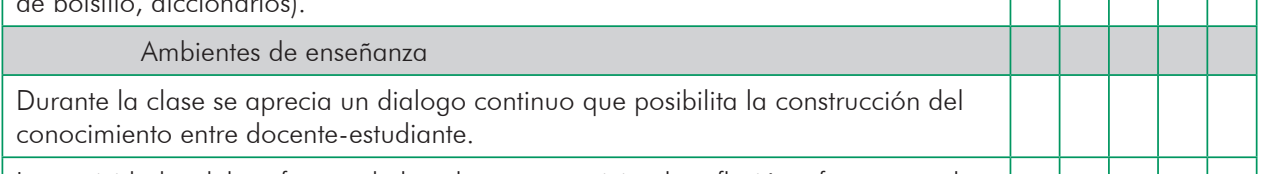

Las actividades del profesor y de los alumnos propician la reflexión y favorecen el aprendizaje de los estudiantes.

Los métodos o estrategias planteadas durante la clase permiten alcanzar los objetivos de la misma.

Los métodos o estrategias planteadas son eficientes en relación al tiempo de enseñanza y aprendizaje.

El profesor logra la participación activa de los estudiantes.

El profesor resalta los aportes, virtudes y cualidades de los estudiantes de manera apropiada.

El profesor utiliza diferentes estrategias o herramientas tales como medios audiovisuales, prácticas de laboratorio, talleres de manera clara que apoyen el desarrollo de la clase de manera efectiva y orientada hacia los objetivos establecidos.

Las actividades en clase permiten a los estudiantes el trabajo y el intercambio en grupo/equipo.

El profesor permite diversos caminos de aprendizaje y hace uso del error como oportunidad de aprendizaje.

El profesor motiva a los estudiantes dispersos a participar activamente en clase.

El profesor aprovecha fases del trabajo autónomo para el apoyo personalizado de estudiantes de manera individual o de grupos.

El profesor motiva y despierta el interés en los estudiantes incluyendo en el desarrollo de las clases las relaciones entre ciencia, tecnología, sociedad y ambiente.

El profesor observa detenidamente a sus estudiantes para determinar su estado de ánimo y posibles problemas que interfieran con su proceso de aprendizaje, para poder ayudarlos en la medida de sus posibilidades.

El profesor identifica las dificultades de aprendizaje que puedan tener los estudiantes y las toma en consideración para el diseño de sus actividades y la implementación de nuevas estrategias en función de poder superarlas.

El profesor en el desarrollo de su práctica integra los contenidos conceptuales, procedimentales y actitudinales del conocimiento científico.

El profesor interrelaciona e integra el conocimiento pedagógico y el conocimiento científico.

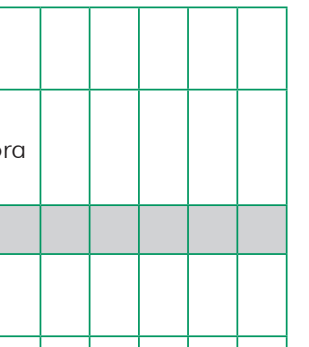

(1)




\section{Escala de valoración}

\begin{tabular}{|l|l|}
\hline \multicolumn{2}{|l|}{ Categoría 1"Estructura de la Clase" } \\
\hline Rango & Valoración \\
\hline $1-9$ & Insatisfecho (IS) \\
\hline $10-20$ & $\begin{array}{l}\text { Parcialmente } \\
\text { Satisfecho (PS) }\end{array}$ \\
\hline $21-30$ & Algo Satisfecho (AS) \\
\hline $31-40$ & Satisfecho (S) \\
\hline $41-50$ & Muy Satisfecho (MS) \\
\hline
\end{tabular}

\begin{tabular}{|l|l|}
\hline \multicolumn{2}{|l|}{ Categoría 2 "Ambientes de Aprendizaje" } \\
\hline Rango & Valoración \\
\hline $1-7$ & Insatisfecho (IS) \\
\hline $8-14$ & $\begin{array}{l}\text { Parcialmente } \\
\text { Satisfecho (PS) }\end{array}$ \\
\hline $15-21$ & Algo Satisfecho (AS) \\
\hline $22-28$ & Satisfecho (S) \\
\hline $29-35$ & Muy Satisfecho (MS) \\
\hline
\end{tabular}

\begin{tabular}{|l|l|}
\hline \multicolumn{2}{|l|}{ Categoría 3 "Ambientes de Enseñanza" } \\
\hline Rango & Valoración \\
\hline $1-15$ & Insatisfecho (IS) \\
\hline $16-30$ & $\begin{array}{l}\text { Parcialmente } \\
\text { Satisfecho (PS) }\end{array}$ \\
\hline $31-45$ & Algo Satisfecho (AS) \\
\hline $46-60$ & Satisfecho (S) \\
\hline $61-75$ & Muy Satisfecho (MS) \\
\hline
\end{tabular}

